\title{
Suboptimal Gradient Artifact
}

National Cancer Institute

\section{Source}

National Cancer Institute. Suboptimal Gradient Artifact. NCI Thesaurus. Code C87008.

An artifact resulting from static and dynamic field changes in a scanner magnetic field. 